\title{
Vortex anomaly in low-dimensional fermionic condensates: Quantum confinement breaks chirality
}

\author{
Yajiang Chen, ${ }^{1,2}$ A. A. Shanenko,,${ }^{2,3,{ }^{*}}$ and F. M. Peeters ${ }^{2}$ \\ ${ }^{1}$ Department of Physics, Lishui University, 323000 Zhejiang, People's Republic of China \\ ${ }^{2}$ Departement Fysica, Universiteit Antwerpen, Groenenborgerlaan 171, B-2020 Antwerpen, Belgium \\ ${ }^{3}$ Departamento de Física, Universidade Federal de Pernambuco, Cidade Universitária, 50670-901 Recife, Pernambuco, Brazil
}

(Received 12 September 2013; revised manuscript received 4 February 2014; published 19 February 2014)

\begin{abstract}
Chiral fermions are responsible for low-temperature properties of vortices in fermionic condensates, both superconducting (charged) and superfluid (neutral). One of the most striking consequences of this fact is that the core of a single-quantum vortex collapses at low temperatures, $T \rightarrow 0$ (i.e., the Kramer-Pesch effect for superconductors), due to the presence of chiral quasiparticles in the vortex-core region. We show that the situation changes drastically for fermionic condensates confined in quasi-one-dimensional and quasi-two-dimensional geometries. Here quantum confinement breaks the chirality of in-core fermions. As a result, instead of the ultimate shrinking, the core of a single-quantum vortex extends at low temperatures, and the condensate profile surprisingly mimics the multiquantum vortex behavior. Our findings are relevant for nanoscale superconductors, such as recent metallic nanoislands on silicon, and also for ultracold superfluid Fermi gases in cigar-shaped and pancake-shaped atomic traps.
\end{abstract}

DOI: 10.1103/PhysRevB.89.054513

PACS number(s): 74.78.Na, 74.25.Ha, 74.25.Uv, 74.81.-g

\section{INTRODUCTION}

Chiral fermions are responsible for many unusual phenomena, ranging from high-energy to condensed-matter physics. Though such fermions were originally introduced in relativistic field theory, it seems hardly an exaggeration to say that their role in present solid-state studies is even more important. The whole realm of graphene physics is largely based on the presence of Dirac points [1]. The same can be said for topological insulators [2]. In superconductivity chiral fermions recently attracted much attention in the contest of Majorana excitations [3]. Yet, chiral quasiparticles localized in the vortex core have been known for type-II superconductors and for rotating fermionic neutral superfluids for more than 50 years $[4,5]$.

Caroli et al. [6] were the first to find that there exist fermionic quasiparticles localized in the vortex core with energies lower than the excitation gap deep in the superconducting state $\Delta_{0}$ (so-called in-gap or in-core fermions). The formation mechanism of such quasiparticles, i.e., Andreev reflection, was revealed through a semiclassical consideration by Andreev [7]. With advances in the experimental technique, those in-gap fermions were eventually observed in the tunneling spectra of type-II superconductors [8].

Though it was clear that in-core quasiparticles should alter the profile of the order parameter $\Delta(\mathbf{r})$, the theoretical study of this issue by Kramer and Pesch [9] produced a striking result. It was revealed that the core of a single-quantum vortex in a clean $s$-wave superconductor collapses at near-zero temperatures. Similar behavior was also found for superfluid fermionic condensates [5], such as atomic Fermi gases [10] and superfluid neutron star matter [11]. The origin of the Kramer-Pesch collapse is the chirality of in-core fermionic excitations [5], whose energy is positive and proportional to the angular momentum $m$. Since the energy goes to zero for $m \rightarrow 0$, fermions localized in the vortex core create a kind of zero-energy Fermi surface [5]. As a result, the

\footnotetext{
*arkady.shanenko@ua.ac.be
}

occupation of such fermionic states changes abruptly when $T \rightarrow 0$. Therefore, in the nearest vicinity of the vortex center, $\Delta(\mathbf{r})$ exhibits a sharp increase over a rather short spatial scale governed by $\xi_{1}=\xi_{0}\left(T / T_{c}\right)$, where $\xi_{0}$ is the usual zerotemperature coherence length and $T_{c}$ is the critical temperature [5,10-12].

In the present work we show that the physical situation changes drastically when a fermionic condensate is confined in quasi-one-dimensional or quasi-two-dimensional geometry. Quantum confinement breaks the chirality of in-core fermions and, instead of the Kramer-Pesch shrinking, the vortex core exhibits an anomalous extension at near-zero temperatures. For illustration, we perform numerical studies of a vortex with a winding number, $n=1$, located in the center of a superconducting nanocylinder. Such ultimate confinement is not only a theoretical possibility: experimental results on a single vortex confined in a flat metallic nanoisland have become available recently [13]. Furthermore, our finding is also relevant to recently realized fermionic condensates in cigar-shaped and pancake-shaped atomic traps [14], where the solid control of the system geometrical parameters makes it possible to observe the predicted anomaly with a high accuracy (see the discussion in Sec. IV).

The present paper is organized as follows. In Sec. II we outline the Bogoliubov-de Gennes (BdG) formalism for a superconducting nanocylinder and then discuss the system parameters and a simple model for taking into account surface roughness. Section III presents our main results. The summary and relevant discussions are given in Sec. IV.

\section{FORMALISM}

Our analysis invokes a numerical solution of the BdG equations [4] designed for an $s$-wave superconducting nanocylinder with a vortex line inside (winding number $n=1$ ). The BdG equations read

$$
\left(\begin{array}{cc}
\hat{H}_{e} & \Delta(\mathbf{r}) \\
\Delta^{*}(\mathbf{r}) & -\hat{H}_{e}^{*}
\end{array}\right)\left(\begin{array}{c}
u_{v}(\mathbf{r}) \\
v_{v}(\mathbf{r})
\end{array}\right)=E_{v}\left(\begin{array}{c}
u_{v}(\mathbf{r}) \\
v_{v}(\mathbf{r})
\end{array}\right),
$$


where $E_{v}$ is the quasiparticle energy, $u_{v}(\mathbf{r})$ and $v_{v}(\mathbf{r})$ are the electronlike and holelike wave functions, respectively, and the single-electron Hamiltonian (measured from the chemical potential $\mu)$ is $\hat{H}_{e}=\frac{1}{2 m_{e}}\left[-i \hbar \nabla-\frac{e}{c} \mathbf{A}\right]^{2}-\mu$, with $m_{e}$ being the effective carrier mass (set to the free electron mass below). We consider the deep type-II regime, which allows us to neglect the magnetic field, i.e., $\mathbf{A}=0$.

The pair potential of a single vortex with winding number $n=1$ is expressed in cylindrical coordinates as $\Delta(\mathbf{r})=$ $\Delta(\rho) e^{-i \theta}$. Consequently, $u_{v}(\mathbf{r})$ and $v_{v}(\mathbf{r})$ can be chosen in the following forms:

$$
\begin{aligned}
& u_{v}(\mathbf{r})=\frac{1}{\sqrt{2 \pi L}} u_{j m k}(\rho) e^{i(m-1 / 2) \theta} e^{i k z}, \\
& v_{v}(\mathbf{r})=\frac{1}{\sqrt{2 \pi L}} v_{j m k}(\rho) e^{i(m+1 / 2) \theta} e^{i k z},
\end{aligned}
$$

where $v=\{j, m, k\}, j$ is the radial quantum number, $m$ is a half-odd integer $[6,15], k$ is the wave number for the longitudinal motion in the $z$ direction, and $L$ is the length of the cylinder. The transverse quantum confinement dictates that $u_{j m k}(R)=v_{j m k}(R)=0$, where $R$ is the cylinder radius. Then, the radial particlelike and holelike wave functions are expanded in terms of the Bessel functions as

$$
\begin{aligned}
& u_{j m k}(\rho)=\sum_{i} c_{j m k, i} \phi_{i, m-\frac{1}{2}}(\rho), \\
& v_{j m k}(\rho)=\sum_{i} d_{j m k, i} \phi_{i, m+\frac{1}{2}}(\rho),
\end{aligned}
$$

with $(\eta=m \pm 1 / 2)$

$$
\phi_{i, \eta}(\rho)=\frac{\sqrt{2}}{R J_{\eta+1}\left(\alpha_{i, \eta}\right)} J_{\eta}\left(\alpha_{i, \eta} \rho / R\right),
$$

where $J_{\eta}$ is the $\eta$ th-order Bessel function of the first kind and $\alpha_{i, n}$ is its $i$ th zero. Plugging Eqs. (2) and (3) into Eq. (1), the $\mathrm{BdG}$ equations are represented in the matrix form so that the key point of the corresponding numerical procedure is to diagonalize the relevant matrix. Here one should take into account the usual normalization condition [4],

$$
\sum_{i}\left(c_{j m k, i}^{2}+d_{j m k, i}^{2}\right)=1,
$$

where $c_{j m k, i}$ and $d_{j m k, i}$ are chosen to be real.

As a mean-field approach, the BdG equations are supplemented by the self-consistency relation

$$
\Delta(\mathbf{r})=g \sum_{0<E_{v} \leq \hbar \omega_{D}} u_{v}(\mathbf{r}) v_{v}^{*}(\mathbf{r})\left[1-2 f\left(E_{v}\right)\right],
$$

with $g$ being the coupling constant, $\omega_{D}$ the Debye frequency, and $f\left(E_{v}\right)$ the Fermi distribution of quasiparticles. The usual way to incorporate Eq. (6) in numerical calculations is to invoke iterations, starting from a proper initial condition [e.g., $\Delta(\rho)=\Delta_{0}$ ] and diagonalizing the corresponding matrix at each step until convergence is reached.

Our interest is to investigate the effects of quantum confinement on the vortex core. Such effects are expected to dominate when the spacing between discrete single-electron levels (here associated with the transverse electronic motion) $\delta \sim \frac{\hbar^{2}}{2 m_{e}} \frac{\pi^{2}}{R^{2}}$ is of the order of or larger than the characteristic pairing energy $\Delta_{0}$. Typically, for metallic parameters we have $\Delta_{0} \sim 0.5-1.0 \mathrm{meV}$, and one reaches $\delta \sim \Delta_{0}$ for $R \sim 30 \mathrm{~nm}$. Consequently, the optimal regime to investigate confinement effects on a single vortex is to adopt $\xi_{0} \sim R \sim 30 \mathrm{~nm}$. For illustration, we choose $\hbar \omega_{D}=30 \mathrm{meV}$ and $\Delta_{0} \approx 1.2 \mathrm{meV}$ (at $T=0$ ), the same microscopic parameters as those used in Ref. [15]. We take the Fermi velocity as $v_{F}=1.4 \times 10^{5} \mathrm{~m} / \mathrm{s}$, which yields $\xi_{0} \approx 25 \mathrm{~nm}$. To simplify and speed up our numerical procedure, we take $k=0$ (as in Ref. [15]), which corresponds to a cylindrical Fermi surface oriented in the $z$ direction. The prototype material can be, e.g., $\mathrm{NbSe}_{2}$ (see Refs. [15-17]).

An unavoidable issue in nanoscale superconductors is nonmagnetic imperfections such as surface roughness or disorder associated with the wetting layer between the superconducting material and the semiconductor substrate. So, it is important to check how the results are stable against such imperfections. The standard procedure to incorporate disorder is to employ the Usadel equations [18]. However, such quasiclassical equations are not useful when analyzing the quantum-confinement effects. So, we stay with the BdG equations and incorporate the disorder in a perturbative manner. Namely, we assume that the single-electron Hamiltonian includes, as a perturbation, an irregular and spatially nonuniform potential that simulates the effects of surface imperfections. Then, to first order in the perturbation, we keep the same wave functions as previously. However, the single-electron levels associated with $\phi_{i, \eta}(\rho)$, i.e., $\epsilon_{i, \eta}^{(0)}=\frac{\hbar^{2}}{2 m_{e}} \frac{\alpha_{i, \eta}^{2}}{R^{2}}$, should be shifted by the perturbation. By analogy with Refs. [19] and [20], we model such a shift by incorporating a noise term in the modified single-electron energies

$$
\epsilon_{i, \eta}=\epsilon_{i, \eta}^{(0)}+s_{i, \eta}\left|\frac{d \epsilon_{i, \eta}^{(0)}}{d R}\right| \delta R,
$$

where $\delta R$ measures fluctuations in the radius and $s_{i, \eta}$ is a random number uniformly distributed in $[-1,1]$. Then, the new energies are incorporated into the matrix $\mathrm{BdG}$ equations to get new self-consistent results. They are subsequently averaged over different random sets of $s_{i, \eta}$.

\section{RESULTS}

Our numerical investigations of the $\mathrm{BdG}$ equations performed for radii $R=30-70 \mathrm{~nm}$ (for the chosen parameters $R / \lambda_{F}=6-12$ ) reveal that quantum confinement of a single vortex results in qualitative changes of its microscopic properties. In particular, we find two different regimes of low-temperature modifications to the vortex core, which we call the ordinary and the anomalous ones. They interchange each time when the radius increases or decreases by approximately $\lambda_{F} / 2$, i.e., when the number of contributing singleelectron levels increases or decreases by one. The ordinary regime is characterized by the Kramer-Pesch shrinking of the vortex core, similar to bulk. This regime is illustrated in Fig. 1(a) by the curve corresponding to $R=60.3 \mathrm{~nm}$. As seen, in the nearest vicinity of the vortex center $\Delta(\rho)$ sharply increases up to $0.4 \Delta_{0}$ on the scale of $\xi_{1} \approx 0.05 R$, 

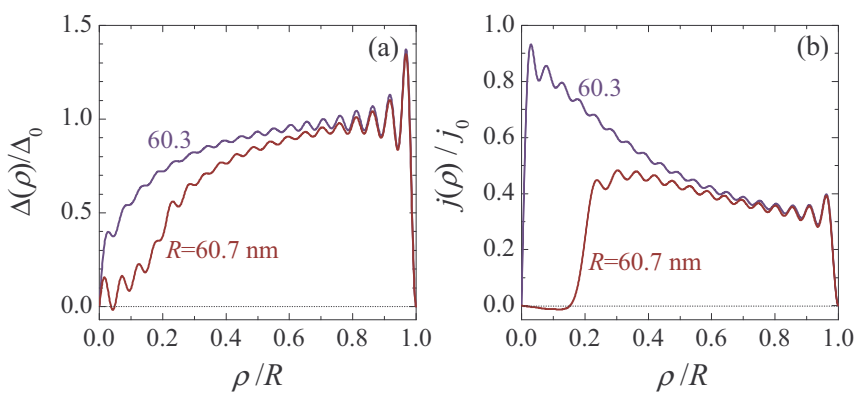

FIG. 1. (Color online) Microscopic vortex characteristics for two different radii, $R=60.3 \mathrm{~nm}$ (ordinary) and $R=60.7 \mathrm{~nm}$ (anomalous): (a) the relative order parameter $\Delta(\rho) / \Delta_{0}$ versus $\rho$; (b) the current density $j(\rho)$ (in units $j_{0}=48 \mathrm{nA} / \mathrm{nm}^{2}$, chosen for our convenience) as a function of $\rho$.

and then gradually approaches $\Delta_{0}$ on the larger scale of $\xi_{0} \approx 0.4 R$. Notice that although our results are calculated for zero temperature, $\xi_{1}$ is small but not zero. The point is that below the quantum limit $T / T_{c}<1 /\left(k_{F} \xi_{0}\right)$, with $k_{F}$ being the Fermi wave number, the discreteness of the in-gap fermionic states starts to manifest itself through the Friedel oscillations of $\Delta(\mathbf{r})$ in the vortex core $[12,21]$. The latter shadow the Kramer-Pesch collapse so that $\xi_{1}$ approaches $\lambda_{F} / 2$ (see, e.g., Refs. [12,21]).

The anomalous regime exhibits a completely different low-temperature trend. In this case the vortex core does not shrink, as illustrated by the data for $R=60.7 \mathrm{~nm}$ given in Fig. 1(a). Instead, the vortex core expands, and the spatial distribution of the condensate near the origin of the coordinates (more precisely, its averaged trend) mimics, to a great extent, the profile of the order parameter in the center of multiquantum vortices [22]. This increase in the size of the vortex core is even more visible in the dependence of the current density on $\rho$ [see Fig. 1(b)]. Notice that, surprisingly, the current density calculated for $R=60.7 \mathrm{~nm}$ even changes its sign in the core region. Because of the combination of pronounced Friedel oscillations with the core expansion, the order parameter can also cross zero for small $\rho / R$. However, this is not always the case, as seen from Fig. 2, where anomalous $\Delta(\rho)$ is shown for radii $R=63.8,67.1$, and $73.5 \mathrm{~nm}$. When the radius increases, the anomalous core expansion becomes less pronounced and

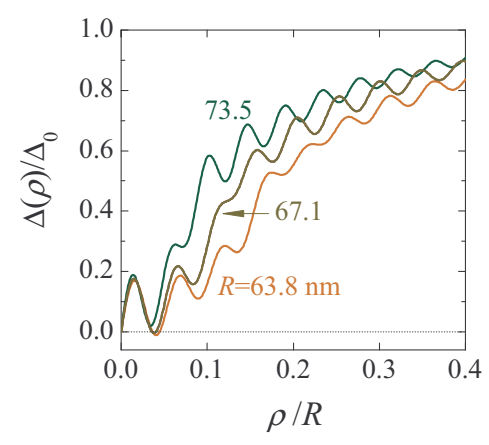

FIG. 2. (Color online) The anomalous regime of the vortex-core behavior for different radii, $R=63.8,67.1$, and $73.5 \mathrm{~nm}$.
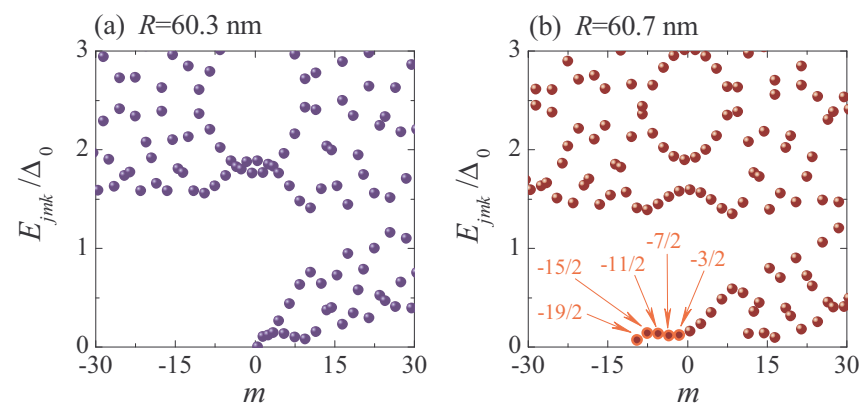

FIG. 3. (Color online) Quasiparticle energy $E_{j m k}$ in units of $\Delta_{0}$ given as a function of the angular momentum $m$ for $R=60.3 \mathrm{~nm}$ (a) and $R=60.7 \mathrm{~nm}(\mathrm{~b})$. Calculations are for zero temperature.

is washed out eventually together with any signatures of quantum-size effects.

Now the question arises as to what physics is behind the breakdown of the Kramer-Pesch effect. The answer is illustrated by Fig. 3, where the quasiparticle energies $E_{j m k}$ are shown versus the angular momentum $m$ for the regular (a) and anomalous regimes (b). In Fig. 3(a) all in-gap fermions $\left(E_{j m k} \lesssim \Delta_{0}\right)$ are chiral; i.e., positive-energy fermions have positive angular momentum $m$. They are mainly located in the vortex-core region and responsible for the Kramer-Pesch effect, in agreement with the standard bulk arguments [5,12]. The situation is qualitatively different in the anomalous regime. As seen in Fig. 3(b), here in-gap excitations with negative $m$ appear. Therefore, the chirality of the in-gap (in-core) fermions is broken, which breaks in turn the Kramer-Pesch shrinking. The explanation is that the states with negative $m$ produce negative contributions to the order parameter. As the corresponding energies are close to zero, these contributions play a significant role only at near-zero temperatures, which is the reason for the core expansion observed in Figs. 1 and 2. The appearance of such negative contributions is a result of depairing caused by interplay between quantum confinement and superfluid motion.

In other words, in addition to the length scales $\xi_{0}$ and $\xi_{1}$, we obtain a new anomalous length which is associated with in-core fermions having negative $m$. Such fermions do not appear in bulk for a single-quantum vortex: they are purely the consequence of the size-quantization effects. So, it is convenient to introduce the length $\xi_{+}=\xi_{1}$, related to the contribution of in-core (in-gap) fermions with positive $m$, and the length $\xi_{-}$, associated with in-core fermions having negative $m$. The breakdown of the Kramer-Pesch effect occurs when the in-core fermions with negative $m$ dominate. This wipes out the length-scale $\xi_{+}$, and the low temperature profile of the vortex core is governed by $\xi_{-}$. As we have the following hierarchy of scales $\xi_{+} \ll \xi_{-} \ll \xi_{0}$, the breakdown of the chirality of the in-core fermions results in a significant expansion of the vortex core.

It is now important to check whether or not our findings are stable against surface roughness and other surface imperfections like a disordered wetting layer between a nanoscale superconductor and the corresponding substrate. For this purpose we add the noise term in the single-electron energy as given by Eq. (7). Figure 4 illustrates the impact 

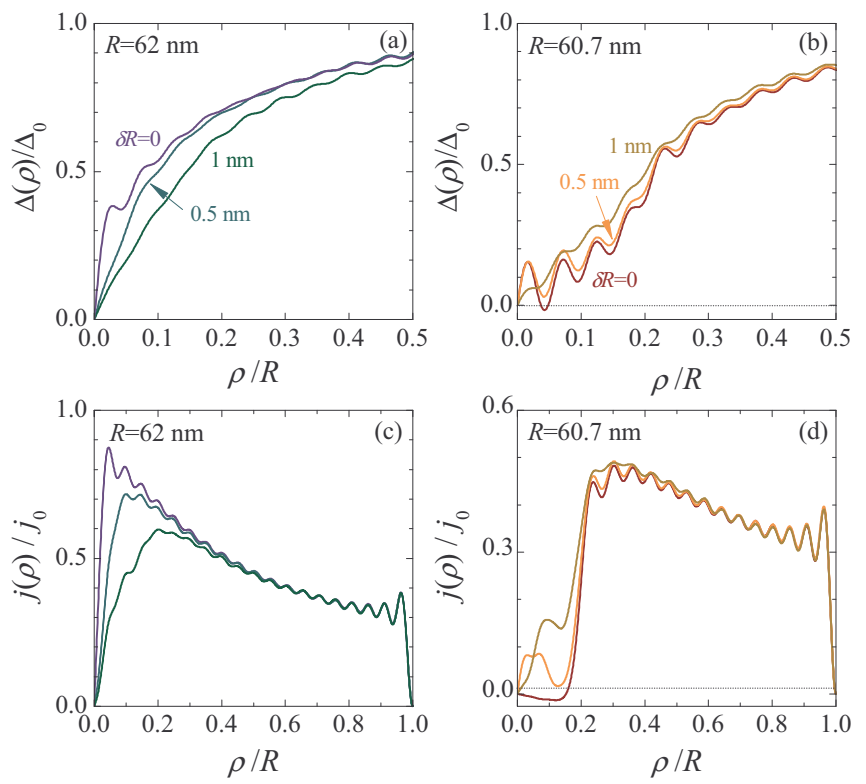

FIG. 4. (Color online) Impact of the surface roughness. Panels (a) and (c) show the data for the order parameter and the supercurrent density for $R=62 \mathrm{~nm}$ in the ordinary regime; panels (b) and (c) represent the anomalous regime for $R=60.7 \mathrm{~nm}$. In each panel the data for the three different fluctuations $\delta R=0,0.5$, and $1.0 \mathrm{~nm}$ are given.

of surface roughness in the case of the ordinary KramerPesch regime [panels (a) and (c)] and the anomalous regime [panels (b) and (d)]. The data are presented for two radii, $R=62 \mathrm{~nm}$ (ordinary) and $R=60.7 \mathrm{~nm}$ (anomalous), and each panel includes curves for three different sizes of the radius fluctuations $\delta R=0,0.5$, and $1 \mathrm{~nm}$ (such fluctuations should be of the order of the lattice constant of the corresponding material). Upper panels demonstrate how the dependence of the order parameter on $\rho / R$ is sensitive to an increase in $\delta R$ while the lower panels are related to the current density. From panels (a) and (c) one can see that the Kramer-Pesch shrinking is rather sensitive to the surface roughness. This fully agrees with the usual expectations that the Kramer-Pesch effect in bulk $s$-wave superconductors is smeared out in the presence of impurities [23,24]. In contrast, the anomalous expansion of the vortex core is significantly more stable. As seen from panels (b) and (d), the changes in $\Delta(\rho)$ and $j(\rho)$ are less pronounced in this case even for $\delta R=1 \mathrm{~nm}$ (i.e., the onset of the steep change in $j(\rho)$ is practically independent of $\delta R)$. On the other hand, the Kramer-Pesch effect is already heavily affected at $\delta R=0.5 \mathrm{~nm}$.

\section{CONCLUSIONS}

In summary, we demonstrated that quantum confinement has a dramatic effect on the microscopic properties of vortices. In particular, a vortex with winding number $n=1$ confined in an $s$-wave superconducting nanocylinder exhibits an anomalous low-temperature expansion of its core rather than the Kramer-Pesch collapse typical of bulk clean superconductors. The physics behind such an anomaly is that quantum confinement breaks the chirality of in-core fermions responsible for the low-temperature core collapse in bulk. We found that the effect is rather stable against surface imperfections, which favors its possible observations in experiments with nanoscale superconductors. Based on our findings we can expect similar results for a vortex in quasi-two-dimensional geometry, such as metallic nanoislands deposited on silicon. Confined fermionic condensates are now also available in experiments with atomic traps [14] where the cigar-shaped and pancake-shaped fermionic condensates have recently been realized. Notice that the shape of the confining potential is not of importance for our study until the coherence length is smaller than the system dimensions. In particular, the effect of interest is due to a pronounced energy spacing between the single-particle levels, and it appears for both "hard-wall" and "soft-wall" potentials. Thus, the "anti"Kramer-Pesch trend can also be observed in ultracold atomic condensates.

We note that the reported oscillations of the vortex core with changing radius (i.e., interchange of the ordinary and anomalous regimes) are a new quantum-size effect in addition to the well-known shape (quantum-size) resonances theoretically investigated in ultrathin films [25], quantum-striped superconductors [26], superconducting metallic nanowires [27], and pancake-shaped and cigar-shaped superfluid Fermi gases [28,29], and recently observed in atomically flat superconducting metallic nanofilms [30]. Thus, we believe that our findings will open a new chapter in studies of superconducting and superfluid quantum-size oscillations in quasi-one-dimensional and quasi-two-dimensional fermionic condensates.

\section{ACKNOWLEDGMENTS}

This work was supported by the "Odysseus" Program of the Flemish Government, the Flemish Science Foundation (FWO-Vl), the Methusalem Program, and the National Science Foundation of China under Grant No. NSFC-11304134. A.A.S. acknowledges the support of Brazilian agencies $\mathrm{CNPq}$ and FACEPE (Grant No. APQ-0589-1.05/08).
[1] A. H. Castro Neto, F. Guinea, N. M. R. Peres, K. S. Novoselov, and A. Geim, Rev. Mod. Phys. 81, 109 (2009).

[2] M. Z. Hasan and C. L. Kane, Rev. Mod. Phys. 82, 3045 (2010).

[3] V. Mourik, K. Zuo, S. M. Frolov, S. R. Plissard, E. P. A. M. Bakkers, and L. P. Kouwenhoven, Science 336, 1003 (2012).

[4] P. G. de Gennes, Superconductivity of Metals and Alloys (Benjamin, New York, 1966).
[5] G. V. Volovik, JETP Lett. 58, 455 (1993) [in Russian, Pis'ma Zh. Eksp. Teor. Fiz. 58, 444 (1993)]; Phys. C 235, 2411 (1994); JETP Lett. 70, 609 (1999) [in Russian, Pis'ma Zh. Eksp. Teor. Fiz. 70, 601 (1999)].

[6] C. Caroli, P. de Gennes, and J. Matricon, Phys. Lett. 9, 307 (1964).

[7] A. F. Andreev, Sov. Phys. JETP 19, 1228 (1964) [in Russian, Pis'ma Zh. Eksp. Teor. Fiz. 46, 1823 (1964)]. 
[8] H. F. Hess, R. B. Robinson, R. C. Dynes, J. M. Valles, and J. V. Waszczak, Phys. Rev. Lett. 62, 214 (1989).

[9] L. Kramer and W. Pesch, Z. Phys. 269, 59 (1974).

[10] N. Nygaard, G. M. Bruun, C. W. Clark, and D. L. Feder, Phys. Rev. Lett. 90, 210402 (2003).

[11] F. V. De Blasio and Ø. Elgarøy, Phys. Rev. Lett. 82, 1815 (1999).

[12] J. E. Sonier, J. H. Brewer, and R. F. Kiefl, Rev. Mod. Phys. 72, 769 (2000).

[13] T. Cren, D. Fokin, F. Debontridder, V. Dubost, and D. Roditchev, Phys. Rev. Lett. 102, 127005 (2009); T. Cren, L. Serrier-Garcia, F. Debontridder, and D. Roditchev, ibid. 107, 097202 (2011).

[14] I. Bloch, J. Dalibard, and W. Zwerger, Rev. Mod. Phys. 80, 885 (2008).

[15] F. Gygi and M. Schlüter, Phys. Rev. B 43, 7609 (1991).

[16] L. F. Mattheiss, Phys. Rev. Lett. 30, 784 (1973).

[17] J. E. Graebner and M. Robbins, Phys. Rev. Lett. 36, 422 (1976).

[18] J. B. Ketterson and S. N. Song, Superconductivity (Cambridge University Press, Cambridge, UK, 1999).

[19] J. E. Han and V. H. Crespi, Phys. Rev. B 69, 214526 (2004).

[20] Y. Chen, A. A. Shanenko, M. D. Croitoru, and F. M. Peeters, J. Phys.: Condens. Matter 24, 265702 (2012).

[21] N. Hayashi, T. Isoshima, M. Ichioka, and K. Machida, Phys. Rev. Lett. 80, 2921 (1998).

[22] S. M. M. Virtanen and M. M. Salomaa, Phys. Rev. B 60, 14581 (1999).

[23] Y. Kato and N. Hayashi, J. Phys. Soc. Jpn. 70, 3368 (2001).
[24] N. Hayashi, Y. Kato, and M. Sigrist, J. Low Temp. Phys. 139, 79 (2005).

[25] J. M. Blatt and C. J. Thompson, Phys. Rev. Lett. 10, 332 (1963); M. Yu, M. Strongin, and A. Paskin, Phys. Rev. B 14, 996 (1976); B. Chen, Z. Zhu, and X. C. Xie, ibid. 74, 132504 (2006); A. A. Shanenko, M. D. Croitoru, and F. M. Peeters, ibid. 75, 014519 (2007); Y. Chen, A. A. Shanenko, A. Perali, and F. M. Peeters, J. Phys.: Condens. Matter 24, 185701 (2012).

[26] A. Perali, A. Bianconi, A. Lanzara, and N. L. Saini, Solid State Commun. 100, 181 (1996).

[27] A. A. Shanenko, M. D. Croitoru, M. Zgirski, F. M. Peeters, and K. Arutyunov, Phys. Rev. B 74, 052502 (2006); A. A. Shanenko, M. D. Croitoru, R. G. Mints, and F. M. Peeters, Phys. Rev. Lett. 99, 067007 (2007); A. A. Shanenko, M. D. Croitoru, A. Vagov, and F. M. Peeters, Phys. Rev. B 82, 104524 (2010); Y. Chen, M. D. Croitoru, A. A. Shanenko, and F. M. Peeters, J. Phys.: Condens. Matter 21, 435701 (2009); Y. Chen, A. A. Shanenko, and F. M. Peeters, Phys. Rev. B 81, 134523 (2010).

[28] J.-P. Martikainen and P. Törmä, Phys. Rev. Lett. 95, 170407 (2005).

[29] A. A. Shanenko, M. D. Croitoru, A. V. Vagov, V. M. Axt, A. Perali, and F. M. Peeters, Phys. Rev. A 86, 033612 (2012).

[30] Y. Guo, Y.-F. Zhang, X.-Y. Bao, T.-Z. Han, Z. Tang, L.-X. Zhang, W.-G. Zhu, E. G. Wang, Q. Niu. Z. Q. Qiu, J.-F. Jia, Z.-X. Zhao, and Q.-K. Xue, Science 306, 1915 (2004); D. Eom, S. Qin, M.-Y. Chou, and C. K. Shih, Phys. Rev. Lett. 96, 027005 (2006). 